\title{
Estar juntos/estar separados en el escenario urbano cordobés actual
}

\section{MARÍA EUGENIA BOITO}

meboito@yahoo.com.ar - Centro de Investigaciones y Estudios sobre Cultura y Sociedad (CIECS), Universidad Nacional de Córdoba (UNC), Argentina

Fecha de recepción: 20 de abril de 2017

Fecha de aceptación: 10 de mayo de 2017

\section{RESUMEN}

Las presentes reflexiones retoman algunos desarrollos de la investigación en curso que realizamos en el marco de la Secretaria de Ciencia y Técnica dela Universidad Nacional de Córdoba, Argentina, titulada "Urbanismo estratégico y conflictos: experiencias de disputas urbanas en Córdoba Capital (2016-2017)”. Hemos indagado en diversos barrios: Alberdi, San Vicente, Güemes, San Martín, El Tropezón, donde ciertos actores luchan por permanecer en el espacio urbano del que han sido/vienen siendo desplazados, realizando demandas de subjetividad eidentidad que se traman en situaciones heterogéneas de conflicto frente al avasallamiento del desarrollismo inmobiliario y de los gobiernos municipal y provincial.

Ante esto, en Alberdi, la Multisectorial; en San Vicente, la Red de Vecinos, los vecinos del asentamiento El Tropezón, delaex-cárceldeSan Martínylosartesanos de Güemes, se constituyen como actores sociales en estos barrios pericentrales. En esta presentación retomamos la idea de M. de Certeau para dar cuenta de algunas tácticas que actualizan los miembros de estos colectivos ylas interrogamos en cuanto a los horizontesdecambiosocialquetramanyexpresan. Elobjetivo es dar cuenta de algunos núcleos centrales de sentido en tanto organizadores de la acción con otros, en el espacio/temporalidad actual de la ciudad cordobesa, en vistas a pensar - junto a J.M. Barbero- sobre algunasmanifestacionesen el presentedel "estarjuntos", construyendo significados sobre el barrio/la ciudad.
PALABRAS ClAVE: Disputas urbanas, Comunicación, Ideología, Clases, Conflictos

\begin{abstract}
The following considerations gather up some of the current research developments we have carried out in the context of the Secretary of Science and Technique of the National University of Córdoba, in a project titled: "Strategic urbanism and conflicts: experiences of urban disputes in Cordoba (2016-2017)".

We have investigated in several neighborhoods of the city: Alberdi, San Vicente, Güemes, San Martín, El Tropezón. In such territories certain actors struggle to stay on the urban grid from where they have been or are being displaced. In the process, they elaborate subjective and identity demands that weave into different conflict situations facing the violence of urban developers and of local and provincial governments.

In such a context, in Alberdi neighborhood rises "The Multisectorial"; in San Vicente, the "Network of neighbors"; people from El Tropezón and nextdoor inhabitants of the former San Martín Prison, such as the Craftsmen of Güemes: these are the main actors in the nearby down town area.

In this presentation, there for, we resume ideas proposed by Michel de Certeau to explain some of the tactics that the members of these collective experiences unfold. We also interrogate these actors and practices about the transformation horizons they express. Our main goal is to present some of the central core meanings that organize actions with others in present space/time of Cordoba city. In this sense, we wish toponder on some of the present manifestations of "being together" - with J.M. Barbero-- building meanings about our neighborhood and city.

KEYWORDS: Urban disputes, communication, ideology, classes, conflicts 


\section{INTRODUCCIÓN}

Las presentes reflexiones retoman algunos desarrollos de la investigación en curso que realizamos en el marco de la Secretaria de Ciencia y Técnica de la Universidad Nacional de Córdoba, Argentina, titulada "Urbanismo estratégico y conflictos: experiencias de disputas urbanas en Córdoba Capital (20162017)".

Proponemos exponer lecturas acerca de la conflictividad latente y manifiesta en relación a la experiencia urbana a partir de la intervención del Estado vía políticas públicas, en concordancia con actores del sector privado de la construcción, fundamentalmente durante de la última década en el escenario cordobés.

Hemos indagado en diversos barrios: Alberdi, San Vicente, Güemes, San Martín, El Tropezón, donde ciertos actores luchan por permanecer en el espacio urbano del que han sido/vienen siendo desplazados, realizando demandas de subjetividad e identidad que se traman en situaciones heterogéneas de conflicto frente al avasallamiento del desarrollismo inmobiliario y de otros actores socio-económicos que actúan en la ciudad.

Ante esto, en Alberdi, la Multisectorial, en San Vicente, la Red de Vecinos, los vecinos del asentamiento El Tropezón, de la ex cárcel de San Martín y los artesanos de Güemes, se constituyen como actores sociales en estos barrios pericentrales. Por ello al inicio de la investigación nos preguntábamos: ¿cuáles son y cuáles características tienen las experiencias de disputas urbanas en Córdoba Capital, a partir de la operatoria de las principales políticas públicas y privadas orientadas a la configuración de entornos clasistas en el habitar/ circular la ciudad?, ¿cómo es que esa geometría impacta en las gramáticas de las acciones entre corporalidades que se ubican diferencial y desigualmente en el espacio?, ¿cuáles sentidos crea y recrea la experiencia social de disputa urbana en dicho contexto?

En esta presentación retomamos la idea de M. de Certeau (2000) para dar cuenta de algunas "tácticas" que actualizan los miembros de estos colectivos y las interrogamos en cuanto a los horizontes de cambio social que traman y expresan, en tanto manifestación ideológica de lo que se configura como objeto de protesta, repertorios de visibilización, formas/contenidos de las demandas, interpelaciones a las audiencias, configuración de antagonistas.

El objetivo es dar cuenta de algunos núcleos centrales de sentido en tanto organizadores de la acción con otros; núcleos como posibilitantes y limitantes de las maneras posibles y deseables de disputa por vivir en la ciudad, que son la resultante de un momento y un estado de las luchas sociales en su reconfiguración socio-histórica, a partir de los logros y las derrotas en este campo de lucha social -y de clases-. 
La identificación de algunos núcleos centrales de sentido en tanto organizadores de la acción con otros, en el espacio/temporalidad actual de la ciudad cordobesa, hace posible pensar -junto a J.M. Barbero- sobre algunas manifestaciones en el presente del "estar juntos", construyendo significados sobre el barrio/la ciudad.

La estrategia expositiva y argumentativa es la siguiente: en primer lugar, realizamos una breve presentación de escenarios barriales, actores, cuestiones que se tematizan como conflictos barriales/urbanos y lógicas de acción que ponen en acto los colectivos; luego, a partir de las recurrencias observadas, proponemos una identificación de núcleos de sentido organizadores del horizonte de cambio social y concretamos interpretaciones sobre las posibilidades de libertad y de restricción de la acción a las que se asocian; finalmente, retomando algunas consideraciones de Jesús Martín Babero sobre desde donde pensamos hoy la comunicación y su perspectiva sobre la misma, conformada por procesos, prácticas y medios a través de los cuales se materializan formas de "estar juntos", pretendemos contribuir a abrir los posibles - y dar cuenta de sus limitantes- en conflictos urbanos que no pueden evitar ser leídos como la manifestación de la operatoria del capitalismo - en su forma de finanzas y de construcción - con formas de despojo sobre espacios y cuerpos según clase.

\section{CONSIDERACIONES TEÓRICAS INICIALES}

En la última década la ciudad de Córdoba experimentó numerosas transformaciones, tanto por cambios en el orden normativo que permite el ingreso creciente de capitales privados en la obra pública como por la efectiva articulación Estado/Mercado en mega emprendimientos. De este modo - y siguiendo la pista de Sennett (1997) - podemos afirmar que las modificaciones en la "piedra" (materia) impactaron fuertemente en cómo los cuerpos interactúan en el espacio social general (la ciudad), tal como venimos registrando en diversas investigaciones. Este tipo de decisiones públicas y privadas intervienen en aquello que identificamos como políticas de embellecimiento estratégico (Benjamin, 2005) y urbanismo estratégico (Boito \& Espoz, 2014), orientadas a rediagramar el espacio según dinámicas de disfrute y consumo, condicionando las prácticas del estar con otros en el espacio-tiempo urbano que se modifica y, fundamentalmente, se fragmenta vertiginosamente.

Acompañando los procesos de reestructuración del capital a nivel global y como forma de gestionar los conflictos asociados a esta dinámica, es posible indicar ciertas intervenciones del Estado cordobés, como el desarrollo de complejos habitacionales socio-segregados por el proceso de embellecimiento estratégico de ciertas áreas. Así, el Estado promueve emprendimientos comerciales y el reacondicionamiento de viejos espacios destinados a la vivienda 
(Barrio Juniors, Alta Córdoba, General Paz, Güemes) o a la industria (como en Alberdi y San Vicente) orientándose al espectáculo (Corredor Centro, Parque de las Tejas, Paseo del Buen Pastor).

$\mathrm{Al}$ respecto, es posible especificar que si el capital encuentra su eficacia en el establecimiento persistente - pero igualmente cambiante- de pautas de circulación de mercancías, cuerpos e ideas, su reverso constitutivo está dado por la posibilidad de suspender/detener ciertos movimientos en el espacio, a través del emplazamiento de muros en la traza urbana, círculos de encierro a los cuales los sujetos se ven conminados y regulaciones sensitivas que definen los modos de establecer las interacciones (como veremos en el caso del asentamiento El Tropezón).

Por esto, buscamos observar las fuerzas expulsógenas y las dinámicas asociadas a la disputa que actualizan ciertos actores sociales para permanecer en los espacios de habitar/transitar/circular. Como hemos indagado hasta el presente, estas transformaciones han generado (ygeneran) formas de resistencia, con diversas direcciones e intensidades (Boito \& Espoz, 2014). Son estos nudos de conflicto los que buscamos referir y caracterizar a partir de la implementación de una estrategia que conecte ciertas expresiones resultantes en cinco barrios delas diversas modalidades deintervención en el espacio-tiempo de la urbe actual.

En Alberdi, San Vicente, Güemes, San Martín y El Tropezón ciertos actores buscan luchan por permanecer en el espacio urbano del que han sido/vienen siendo desplazados, defendiendo las memorias colectivas frente al avasallamiento del desarrollismo inmobiliario y de otros actores socioeconómicos que actúan en el escenario urbano cordobés.

Las siguientes hipótesis organizan nuestra investigación en particular y también son referidas en este escrito: a) las transformaciones físicas, arquitectónicas y "viales" en las ciudades están relacionadas con modificaciones en las experiencias posibles para los sujetos que las habitan; b) en la ciudad de Córdoba dichas transformaciones han tendido a la construcción de entornos clasistas; c) las políticas públicas y privadas, así como los emprendimientos que las materializan, tienen impactos en la configuración de las experiencias de los actores que habitan, viven y circulan en la ciudad; d) dadas las transformaciones urbanas en la última década, emergen expresiones de disputa que merecen ser estudiadas en profundidad; e) las formas emergentes de conflictividad en el campo de las disputas urbanas aparecen como una instancia privilegiada para interrogar los marcos que organizan los posible/deseable en términos de horizontes de cambio social. 


\section{COLECTIVOS SOCIALES Y DISPUTAS URBANAS}

Figura 1. Mapa

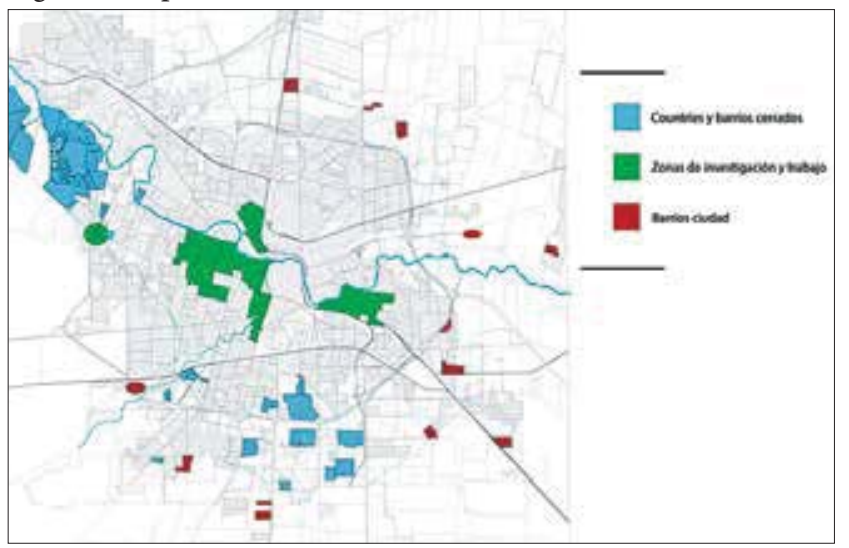

Fuente: Mapa elaborado por el Licenciado Esteban Fernández, FCC, UNC.

\section{Güemes $^{1}$}

Barrio Güemes está ubicado en el sudeste del área central de la ciudad de Córdoba, a 200 metros de la misma. Colinda con el centro, Bella Vista, Observatorio, Nueva Córdoba y Alberdi. Según el censo 2010, 11.000 personas habitan allí.

Cuando se compara la información intercensal en esta zona, más que una pérdida paulatina de habitantes, lo que podemos señalar - en función de lo generado por las investigaciones individuales y colectivas realizadas por nuestro equipo- es la existencia de una clara tendencia de gentrificación. Desde 1980 en adelante, primero se fueron expulsando familias obreras, y posteriormente (1990-2000), familias que habitaban asentamientos: grupos familiares asentados en la zona que fueron desplazados por el gobierno provincial, vía la construcción de barrios-ciudad en la periferia urbana, durante la primera década del 2000. Esto último expresa la fuerza expulsógena de la acción estatal con relación a las clases subalternas y sus posibilidades de habitar un escenario en profunda transformación, que prepara el territorio del barrio tanto como zona turística de bares y paseos de compras, como para emprendimientos inmobiliarios destinados al habitar de otras clases, mediante la construcción en altura. En este sentido, la noción de gentrificación parece pertinente antes que la lectura y fundamentación ideológica que realizan tanto algunos técnicos como investigadores, para los cuales los motivos de la alta intervención urbanística en la zona se fundan en la presencia de importantes áreas en estado de abandono y/o sub-utilización y una creciente pérdida de población.

1 Véase a María Eugenia Boito y Ailen Suyai Pereyra (2016, enero-junio). 
En los inicios históricos de su conformación fue una de las primeras ampliaciones dela ciudad, sin planificación alguna. Durantela intendencia de Luis Revol, a fines de la década de 1880, se realizó sobre la Plaza de las Carretas un complejo de casas para familias de obreros. Sobre este particular espacio - actualmente conocido como El Paseo de las Artes-iniciamos el reconocimiento de la operatoria de la fuerza de patrimonialización que actúa en Güemes desde la última dictadura hasta el presente.

Como señalábamos en otro escrito (Boito \& Espoz, en prensa) la ciudad de Córdoba no es ajena a ciertas modificaciones reguladas por el mercado internacional de bienes materiales e inmateriales que pueden disputarse el estatus de "patrimonio". En la última década - y en el marco de los 5 años posteriores a los festejos nacionales por el Bicentenario de la Patria-, diferentes dimensiones de la vida material, cultural o histórica de "la Docta" (nominación que recibe la ciudad cordobesa por la temprana presencia de la universidad) fueron objeto de tematización/discusión en el marco de lo que podemos reconocer como un fuerte proceso de patrimonialización.

Con el paso de los años, Güemes ha sido clasificado como uno de los barrios tradicionales o pericentrales de la ciudad de Córdoba. En esa dirección, desde la década del '90 el Estado Municipal en conjunto con el Provincial han implementado una serie de planes estratégicos, que buscaron reconstituir el sentido de la imagen de Córdoba Patrimonial, asociándolo al pasado colonial y resaltando su importancia como barrio tradicional. Güemes es una de las áreas de mayor impacto de la reconfiguración sobre el uso del suelo, la creación de un cuerpo normativo y la implementación de "programas o eventos especiales"'.

En la actualidad un hito significativo es la puesta en marcha del "Portal Güemes”. Si bien se firmó un proyecto en el 2009, las primeras obras se realizaron en el 2011 y no finalizaron. El propósito era instalar una especie de "San Telmo cordobés" (en referencia al barrio de Ciudad Autónoma de Buenos Aires), haciendo evidente el modelo al que remiten los sucesivos gobiernos y actores privados, que también se manifiesta en expresiones como "Güemes Soho". A partir de este punto, reconocemos un crecimiento en los "nuevos equipamientos" urbanos - bares y restaurantes-que emergen a partir de sucesivas modificaciones de ordenanzas y creación de nuevas con relación a la ocupación del suelo. Desde 2013 este cambio en Güemes está demostrado con el desembarco de franquicias y de una serie de inversores que apuestan a generar una marca renombrada a nivel mundial: Peñón del Águila, Milo Locket, Havanna, Oye Chico, Venezia son algunos de los ejemplos.

2 Desde el inicio de esta tendencia, la recuperación y la revalorización del patrimonio se va uniendo a la consideración de la cultura como un recurso para potenciar el desarrollo de actividades económicas, en convergencia con servicios turísticos a posteriori y de forma creciente (Boito \& Pereyra, 2016). 
El crecimiento de una variada oferta que ha desbordado el antiguo espacio de la Plaza de las Carretas se expresa en los siguientes datos: para 2013 ya se daba cuenta de una tendencia creciente que se mantiene y se contabilizan 600 artesanos divididos en ferias según rubro y años de antigüedad. Hoy existen ocho ferias y es la denominada Artesanos de La Cañada - considerada "ilegal" porla Municipalidad-aquella dondelos trabajadores, durantelos dosúltimos años, han hecho pública su demanda por permanecer y trabajar en ellugar. Esta tensión tiene por fundamento el ejercicio del derecho a trabajar en este sitio - permanentemente ocupado por turistas de paseo, potenciales compradores/ consumidores - y como horizonte de la acción el ser reconocidos/legalizados por el gobierno municipal.

\begin{abstract}
Alberdi
La ciudad de Córdoba se ha ido transformando en función del crecimiento poblacional, pero también de activas políticas públicas e intervenciones privadas. La expansión de la mancha urbana, la densificación de la población en ciertas zonas, los cambios en la organización económica, socialy tecnológica han reconfigurado las condiciones de habitar, circulary participar en la ciudad. Entre las tendencias hegemónicas se destacan procesos de socio-segregación urbana, fragmentación, gentrificación de barrios tradicionales, privatización dela vida cotidiana y vaciamiento de los espacios públicos; estos cambios se han constituido en objeto de nuestros trabajos de investigación durante la última década (Boito \& Michelazzo, 2015).

Hasta el presente, Barrio Alberdi ha sido objeto de múltiples intervenciones urbanísticas por parte del sector autodenominado "desarrollistas urbanos", que implicaron la construcción de numerosos edificios nuevos y la demolición de construcciones históricas - a pesar de la vigencia de ordenanzas referidas a la protección del patrimonio arquitectónico urbanístico y de áreas de valor cultural-.

En relación a dichos procesos, desde hace varios años, junto a la Multisectorial Defendamos Alberdi (formada en 2010), hemos abordado diferentes aspectos de las transformaciones en el barrio y las maneras en que los mismos afectaban a su población: el impacto sobre aquellos grupos que se han radicado mediante procesos migratorios desde países limítrofes (se trata de un barrio con fuerte presencia de migrantes peruanos), las potencialidades y tensiones con relación a la expropiación municipal del espacio cultural llamando "La Piojera" (antiguo cine-teatro, que en un momento pasó a manos privadas - concretamente era una sede de la Iglesia Universal-) y las intenciones de algunos "vecinos" por potenciar modalidades de "gestión popular" de la misma, el freno ante el avance de grupos económicos que se tradujo y se traduce en la expulsión de las poblaciones más pobres y en la destrucción del patrimonio arquitectónico (por ejemplo, la Casa de
\end{abstract}


la Reforma, donde se gestó la acción reformista de los estudiantes universitarios en $1918^{3}$ ).

De este modo el hecho de constituirse como una zona atractiva para los inversores, ser un barrio con un importante patrimonio histórico cultural de la ciudad y tener una población heterogénea tanto en términos étnicos como de clases sociales, se traduce en diversas expresiones de conflictividad, en función de las múltiples y coexistentes experiencias de lucha.

La Multisectorial - al igual que la Red de Vecinos de San Vicente, como veremos a continuación - se formó por la resistencia a un acontecimiento emergente: el “¡Alto!”, “¡Ya Basta!” a la demolición. En el caso que nos ocupa en este apartado, fue la oposición a la demolición de la chimenea de la Ex Cervecería Córdoba, en 2010 (cervecería cerrada, que antes fue objeto de lucha obrera para evitar el cierre y que hoy constituye un emprendimiento inmobiliario - llamado Antigua Cervecería - que hasta volvió a rehacer como ícono la chimenea demolida y ahora estetizada); mientras que el caso de San Vicente, fue la resistencia a la demolición de la casona del Sindicato de Conductores de Trenes: La Fraternidad, en 2009.

Sin embargo, el desarrollo inmobiliario ha sido desigual entre ambos barrios: en Alberdi el avance de la ciudad "embellecida" es mucho más notorio que en San Vicente ${ }^{4}$. Barrio Alberdi presenta una continuidad geográfica intensa con la zona céntrica: todas las calles que corren de este a oeste por el centro de la ciudad llevan hacia el barrio. Pero, además, la avenida Colón es un corredor que conecta el centro con la zona oeste y noroeste; área de gran crecimiento poblacional y de desarrollo de emprendimientos habitacionales y comerciales para otras, así como camino a "countries" y barrios cerrados de distinto tipo ${ }^{5}$.

Lo antes dicho muestra que la "puesta en valor", la "recuperación" del patrimonio, comola "revitalización” y "recuperación” del río Suquía que atraviesa el barrio, han implicado en muchos casos un activo control sobre la circulación de los miembros de clases subalternas. En los entornos "embellecidos", los habitantes y trabajadores ven restringida sus posibilidades de habitar, trabajar, desplazarse y deben disputar su legitimidad, así se trate del lugar donde han vivido por décadas.

3 "Casa de la Reforma Universitaria. Pasaje Verna 154. Fue demolida por dentro y sólo queda su fachada. Le quitaron una placa identificatoria y desocuparon a quienes vivían allí (era una pensión estudiantil). Levantarían un edificio en sulugar. Otras casonas cercanas vinculadas con la Reforma correrían la misma suerte". Véase:http://www.lavoz. com.ar/ciudadanos/alberdi-alerta-progresiva-desaparicion-inmuebles-historicos

4 En Alberdi, las dos experiencias públicas de expropiación que hubo en el barrio -que en una primera lectura aparecen como un logro de la resistencia y la lucha vecinal- (un cine teatro a manos de la Municipalidad y un conjunto de terrenos públicos ocupados por vecinos pobres literalmente rodeados por las nuevas inversiones, que aunque ahora son "dueños de la tierra" se van a ver obligados a vender) están ubicadas en un territorio donde ha avanzado la demolición "desarrollista": Gama, GNI, Euromayor (antigua cervecería) Proaco Altos de Zípoli (Alberdi), entre otros grupos. Por lo dicho, los "logros" de las expropiaciones estudiadas revelan sus fallas o por lo menos ambigüedades y se inscriben conflictivamente en el estado actual de las relaciones entre Estado y Mercado.

5 Veremos la relación de esto con la construcción del nudo vial El Tropezón. 
En la búsqueda de la defensa de su identidad barrial y memoria colectiva, muchos de estos actores individuales y diversas organizaciones han conformado la "Multisectorial" en Alberdi, con el objeto de disputar las formas de valorizar los espacios intervenidos y dar un sentido distinto al patrimonio. La voluntad colectiva por participar de las decisiones que afectan al barrio se complementa la lucha simbólica por el reconocimiento público y por sumar vecinos a la participación, como anverso dela posición de espectador/consumidor propia de la forma de vida privatizada que se propone desde la imagen de ciudad hegemónica.

\section{San Vicente}

Los barrios de San Vicente y Alberdi han cambiado como partes de un todo que - lejos de ser exclusivos de la ciudad de Córdoba- exponen el lugar de lo urbano como la espacio/temporalidad para la reproducción ampliada del capital (Harvey, 2004) ${ }^{7}$. Como ya veíamos en el abordaje de barrio Alberdi, la lógica del patrimonio encuentra coincidencias profundas con la racionalidad del turismo (en la que también coinciden Estado/Mercado) y en cierta manera puede ser interpretada - con relación a ciertas transformaciones urbanascomo la materialización de una visión museística de la cultura, que prioriza "pasado/conservación" antes quela "creatividad del presente cultural"; es decir, las artes de hacer de las mayorías - sensu De Certeau-que se instancian en este terreno, mediante maneras de hacer cultural heterogéneas y múltiples que actualizan los habitantes de los barrios.

En ambos escenarios barriales el reordenamiento hegemónico intenta imponer nuevas formas de habitar. La expresión más clara es la destrucción de edificaciones tradicionales (casonas, fábricas y otras), y con ellas el borramiento de las condiciones materiales que permitían ciertas maneras de significar y habitar el barrio, así como la presencia de ciertos actores/clases que van siendo desplazados. Sobre estas demoliciones de lo "tradicional", se construyen unidades habitacionales para clases altas o espacios de consumo que modifican las maneras de vivir/sentir/habitary tramar las memorias individuales y colectivas con las vivencias del presente. Los cambios clasistas para la reproducción del

6 Véase a Boito \& Myers (2015) y (2015a, diciembre).

7 "Córdoba se ha convertido en la cuna de una movida desarrollista que supera a otras plazas del país. La actividad duplica a Rosario y crece más que en Buenos Aires, sus empresas ofrecen financiamiento y algunas, incluso, 'exportan' sus proyectos a Uruguay (...). Luego de 2008, el campo dejó de ser el gran financiador de la construcción privada; esto obligó a buscar sectores de la clase media necesitados de vivienda beneficiados por el crecimiento de la economía. (...) Otro factor fue el elevado precio que tomó la tierra en Nueva Córdoba. Además de la poca tierra que queda, el costo atentó contra la rentabilidad de los desarrollistas y obligó a buscar alternativas en otras zonas que permitieran recuperar la rentabilidad. Gracias a la concertación, hoy crecen zonas postergadas de la ciudad", advirtió Carranza, director de la Asociación Española de Instituciones de Inversión Colectiva y Fondos de Pensiones (INVERCO), en una reciente nota publicada por el diario La Voz. Asimismo, expuso que "la participación de la construcción en el empleo del Gran Córdoba el del 10,1 \%, cuando la industria representa 9,2 \%, según un informe de la Ceduc y MKT. El potencial del sector para reducir el desempleo provincial es de $13,1 \%$, cuando en la industria es de 9,3\%. De todas maneras, participa con 11,6\% en el PGB de Córdoba Capital, frente al 15\% de la industria”. Diario La Voz, 24/4/2017. Véase: http://www.lavoz.com.ar/cordoba/cordoba-cuna-desarrollistas. 
capital son materiales; se objetivan en los edificios que se derrumban o construyen y fundamentalmente en la naturaleza sensible de las prácticas de los sujetos que viven o dejan de vivir en ellos, en los haceres y sentidos de quienes habitan y circulan. Es, a fin de cuentas, la resemantización de la vida social lo que está en disputa; por esto es interesante indagar sobre las maneras de tramar el “estar juntos", en la perspectiva de Jesús Martín-Barbero, que empezaremos a explorar en el último apartado.

En comparación con Alberdi, tanto por ubicación como por la propia historia y dinámica social del presente barrial, San Vicente presenta un desarrollo inmobiliario significativamente menor. Estructuralmente sólo se conecta con el centro por la confluencia del Boulevard Perón y la calle Agustín Garzón. Esta área fue fuertemente intervenida por el Estado y el Mercado desde 2004: por el desalojo de Villa La Maternidad y la construcción de la Nueva Terminal de Ómnibus, además de complejos comerciales y habitacionales. Además, como corredor de circulación, San Vicente lleva a barrios humildes y villas de Córdoba, a diferencia de Alberdi.

A pesar de las diferencias, en los escenarios barriales que venimos refiriendo las tendencias hegemónicas de embellecimiento estratégico y la constitución de entornos clasistas $^{8}$ son procesos que están sucediendo y que instauran también las resistencias. En San Vicente se conformó en 2009 la Red de Vecinos y Asociaciones de San Vicente. Entre sus reclamos prima el reconocimiento del valor histórico de lo que llaman "barrios pueblo", la conservación y el mejoramiento de los edificios patrimoniales y la inversión pública en infraestructura en general.

La Red de Vecinos y Asociaciones de San Vicente está constituida fundamentalmente por profesionales (politóloga, arquitecto, psicólogos y otros) y la mayoría residen en la zona. Como decíamos, la Red está ligada a la lucha en defensa de la Casa de La Fraternidad, una casa de ferroviarios que además de su valor arquitectónico, ha constituido un lugar de encuentro entre los vecinos sanvicentinos, para la realización de las más diversas actividades culturales.

Desde la Red se invita a participar a los vecinos en defensa de tal espacio, pero paralelamente se cuestiona constantemente ¿quién es el vecino? Para algunos de sus miembros el vecino sanvicentino, propiamente dicho, no está presente en estas instancias organizativas; para otros el vecino reside en actores colectivos que participan de espacios de la Red (estudiantes de escuelas secundarias, universitarios, quienes participan de la radio comunitaria "la Quinta Pata", Red Ciudadana, etc.). Algunos actores viven en el barrio y otros no - situación similar a la de Alberdi-, pero desde la perspectiva de los miembros de

8 La noción de embellecimiento estratégico remite a las consideraciones de W. Benjamin sobre la ciudad de París, fundamentalmente tras las intervenciones de Haussmann. La noción de entorno clasista es de nuestra autoría junto a la de círculo de encierro, para dar cuenta de los recorridos circulares por clase en función de las transformaciones socio-urbanas que venimos reconociendo en nuestra ciudad. 
la Red ambos incrementan la legitimidad del espacio, y su presencia potencia el establecimiento de redes con otros sujetos, grupos y/o organizaciones con demandas coincidentes.

Otro aspecto en el cual todos están de acuerdo es la importancia de diferenciarse del Estado, principalmente de la Municipalidad, ya que el lugar de encuentro es una plaza de San Vicente, que se ubica al lado del Centro Cultural, que es parte de la administración municipal. Con relación a los centros vecinales, es importante señalar que en los últimos tiempos algunos de los colectivos han iniciado procesos de lucha política en estos espacios, configurando un espectro de posiciones heterogéneas: así por ejemplo, La Multisectorial hoy está en el Centro Vecinal mediante elecciones, en barrio San Vicente se hace patente esta separación/distinción con el Centro Vecinal y en barrio San Martín, el Movimiento Cultural de Barrio San Martín, se ha mantenido generalmente en tensión con esta instancia, como veremos en el próximo apartado.

Cabe aclarar quela Red hace tiempo tenía mayor participación en el Centro Cultural, particularmente en un espacio que llaman "el museíto". El museíto para las autoridades municipales, al no tener una "figura legal", no es reconocido como tal. A lo largo de los años, él mismo ha sido sostenido principalmente por otro empleado municipal con quien los miembros de la Red han trabajado durante muchos años en tal espacio y mantienen una buena relación. Desde la red se realizan visitas guiadas al barrio, donde las construcciones que permanecen remiten a la espacio/temporalidad obrera de décadas atrás. Por ejemplo: las edificaciones de la ex Panadería La Esperanza, los Hornos de Cal Cerrano, Ex Molinos Letizia, el Barrio Obrero Kronfuss, la Casa de Hierro.

En una primera comparación de las tres realidades barriales, señalamos que frente a la especulación y a la conservación museística/turística que orienta las políticas estatales y privadas, en Alberdi y en San Vicente los habitantes de estos barrios cordobeses intentan detener el avance desarrollista en general, pero también en sus emergencias puntuales - como en el caso de la casa de La Fraternidad y la chimenea de la Cervecería - intentan habitarlos como los barrios-pueblo que han sido quieren conservar sus vínculos con el espacio desde la fijación identitaria con un modo de vivir y un pasado común. El reclamo hace énfasis en el valor de uso de la tierra, la vivienda y los espacios comunes, y no en su valor de cambio. La situación en Güemes es otra: su cercanía al centro, los sucesivos desplazamientos de los pobladores y su constitución presente en términos de lugar turístico indican otros conflictos - por ejemplo, la demanda de poder trabajar por parte de algunos de los numerosos artesanos y vendedores que se van ubicando en la peri-feria dela Feria delas Artesanías-. Pero retomando lo antes dicho, el clásico para valor de uso/valor de cambio aparece como significativo para interrogar algunos conflictos y resistencias en Barrio San Martín. 


\section{San Martín}

El Movimiento Cultural de Barrio San Martín (MCBSM) es la instancia vecinal que expresa sus demandas urbanas con relación al barrio y concretamente con la cárcel - hoy vacía - que se ubica en este barrio ${ }^{9}$. San Martín se ubica al noroeste del centro histórico de Córdoba, cruzando el río y sobre las barrancas norte. Ana Clarisa Agüero, basándose en las investigaciones históricas de Waldo Ansaldi y de Cristina Boixados, concreta una breve reseña barrial que seguiremos como guía. Hace 100 años atrás, en términos materiales, existían en lo que hoy es barrio San Martín, las siguientes construcciones:

El molino Ducasse, la Penitenciaría — cuya construcción se había iniciado en 1889-, el Corralón municipal, una fábrica de fósforos (ambos identificados por Boixadós ya en 1889) y, hacia el norte, otra de las construcciones que devendría icónica del barrio: la Cervecería Río Segundo, levantada en 1907 y adyacente a las vías del ferrocarril (...). Es probable que el predio de la Penitenciaría alcanzara temprano sus límites actuales, ya que, aunque los planos contemporáneos registran la construcción en uno de cuatro manzanas, Boixadós releva una donación al gobierno provincialde un terreno conexo entre 1888 y 1890. A ese equipamiento urbano del Pueblo San Martín, seguramente requerido de precisiones y agregados, vendría a añadirse hacia los años veinte otro hito de interés: la llamada Casa Cuna, sobre Castro Barros ${ }^{\mathbf{1 0}}$.

En esta zona habitaban obreros vinculados a las fábricas antes referidas: molineros, cerveceros, ferroviarios, empleados penitenciarios que pudieron acceder a la tierra en función del bajo costo, como ocurrió en otros Barrios-Pueblo. En la actualidad, es evidente el presente como ruina de aquel espacio/tiempode un escenario barrial obrero, confábricas abandonadas, deterioro de la infraestructura, entre otras expresiones.

Tomado el ícono que nuclea la acción colectiva barrial — la Unidad Penitenciaria número 1 (UPI) - , Córdoba no es ajena a lo que ocurre en materia de "reciclaje" y "estetización" de estas construcciones. Como en otras ciudades, las cárceles devienen objeto de transformación estético/política, en función de la rediagramación de los espacios urbanos, que se orientan a desplazar poblaciones que habitan territorios que devienen valiosos en términos de valores de cambio. Así como en el centro de la ciudad de Córdoba - también en la ciudad de Montevideo, Uruguay - una cárcel deviene "Paseo del Buen Pastor" en el primer caso y se transforma en Shopping en el segundo, son vari-

9 No nos hemos referido antes a este tópico, pero la cuestión de la visibilidad en las redes de los colectivos es una preocupación y una ocupación de sus miembros en el marco de la tematización de la comunicación como un "problema" que suele explicar la falta o la insuficiente participación tanto del vecino como del resto dela ciudadanía, en la perspectiva de estos actores. Es así que los diversos colectivos tienen su página en Facebook. Menos en el caso de la comisión de vecinos de El Tropezón -aunque sílos jóvenes, "Los Wachos del Trope"-. Para los vecinos mayores, la producción y circulación de la comunicación sobre sus demandas se ancla y enmarca en el escenario de la villa. Esto se retomará en el próximo apartado. En línea: https://es-la.facebook.com/Movimiento-Cultural-Barrio-Sa n-Mart\%C3\%ADn-579667708838965/

10 Agüero, Ana Clarisa (s/f). En línea: http://culturasinteriores.ffyh.unc.edu.ar 
adas las antiguas formas arquitectónicas típicas de la sociedad disciplinaria de masas que son modificadas para los consumidores/turistas, en este tiempo del capitalismo financiero espectacular - sensu Debord-y de las sociedades de control - sensu Deleuze-. Los diversos escenarios de disciplinamiento de las masas (fábricas, escuelas, cárceles) son estetizados — a veces mediante la conservación dela fachada, como forma de compromiso conla intencionalidad patrimonial-y se transforman en lugares - siempreiluminados ${ }^{11}$ - einscriptos en el recorrido turístico destinado a consumidores/clientes, que atraviesan efímeramente esos espacios. Güemes también tiene la Cárcel de Encausados en los límites del territorio, que persiste cerrada y que ha sido objeto de competencias y premios sobre propuestas de planificación y diseño de sus modificaciones. Mientras que la cárcel de San Martín es lo que ha motorizado y sigue siendo un objeto de movilización de los vecinos y del colectivo. Pero antes de tratar este tema, consideramos necesaria una óptica de mayor alcance - espacial ehistórico-quehaga posible identificar una cruel dinámica socio/urbana de desplazamiento y emplazamiento de las poblaciones, en términos clasistas.

Como hemos indagado en otras instancias (Levstein \& Boito, 2009; Boito \& Espoz, 2014), desde la primera década del 2000 las poblaciones más pobres fueron expulsadas por fuera de ejido de la ciudad, en el marco de programas habitacionales implementados por el gobierno provincial cordobés. Pero no sólo se desplazó y se desalojó a los más pobres que "están en libertad", sino que quienes están en lascárceles dela miseria queha estudiadoL. Wacquant, también fueron expulsados hacia las afueras. En este sentido Bouwer es sintomático: allí se construyeron cárceles y también es la zona de la derivación de los residuos de la ciudad. Hacia la periferia van los cuerpos y los objetos desechables, hacia lugares materiales de encierro como las cárceles, pero también hacia zonas de encierro a puertas abiertas a cielo abierto, como en el caso de los pobladores de las clases subalternas que han sido "beneficiarios" del Plan Mi Casa, Mi Vida", quienes parecen portar una especie de tobillera invisible pero real, ya que están fijados a esos territorios en sus posibilidades de trabajar, circular, interactuar, a partir de la implementación de políticas de seguridad, pos-asesoramiento del Manhattan Institute, al que recurre el gobierno provincial desde 1999.

11 Véase la siguiente nota, que potencia la interpretación dellugar delaluz/iluminación en ciudades que van mutando como ciudades turísticas. Para enfatizar el cambio: si en el momento de la conformación de los estados nacionales primero y de la sociedad de masas luego, de lo que se trataba era de construir grandes edificaciones para la organización "racional"/disciplinada de la vida social, en el presente se trata de iluminarlos, como una forma también de museo a cielo abierto. La iluminación también aparece fuertemente referida en cuestiones de seguridad. En línea: http://www.plataformaurbana.cl/archive/2017/04/01/opinion-la-luz-como-patrimonio-sensorial

12 Luego de las inundaciones que produjo el desborde del Río Suquía y que afectaron a los pobladores que vivían precariamente alrededor de las orillas del mismo, el Gobierno Provincial dispuso el traslado compulsivo de las villas y asentamientos que había en el lugar hacia zonas alejadas del centro de la ciudad y en espacios denominados "ciudadesbarrios". Los complejos habitacionalesson: 1) CiudadEvita (574viviendas), 2) Ciudad de Mis Sueños (565 viviendas), 3) 29 de mayo- Ciudad de los cuartetos (480), 4) Ciudad de los niños (412), 5) Ciudad Obispo Angelelli (359 viviendas), 6) Ciudad Ampliación Ferreyra (460), 7) Ciudad Juan Pablo II (359), 8) Ciudad Villa Retiro (264); 9) Ciudad Parque las Rosas (312), 10) Ciudad Ampliación Cabildo (570), 11) Bo Renacimiento (223), 12) Bo San Lucas (230), 13) Ciudad de mi esperanza (380), 14) Ciudad Villa Bustos (197), 15) Ciudad Sol Naciente (638) 
Volviendo a San Martín, el Movimiento Cultural de Barrio San Martín (MCBSM) es la instancia vecinal que ha organizado 10 "Penal-azos", en tanto espacio/tiempo en el barrio donde, en convergencia con estudiantes y cátedras dela UNC (arquitectura, comunicación, etc.) se realizan actividades culturales para informar al "vecino" sobre el destino planificado por la provincia con relación a las modificaciones y los usos de la cárcel, como también para frenar y oponer otras apropiaciones. La heterogeneidad y la multiplicidad de las demandas mediante las cuales el colectivo pretende interpelar y articular la participación vecinal, dan cuenta tanto de las necesidades presentes del barrio, en función de la falta de inversión y mantenimiento de la infraestructura básica y social por parte de provincia/municipio, como de la voluntad de configurar audiencias y luego participantes de las acciones que propone el grupo. Por ejemplo, el NO a la demolición de la cárcel, la lucha por su reconocimiento en términos patrimoniales se acompaña con las más heterogéneas fundamentaciones: desde la no demolición de la capilla porque allí dio misa el primer santo argentino (Cura Brochero), la utilización del hospital de la cárcel como dispensario para el barrio, el uso de los espacios para resguardo de las mujeres que sufren violencia de género, locales comerciales y la constitución de un sitio de la memoria, ya que por ella pasaron tanto detenidos/desaparecidos de la última dictadura como presos comunes, que desde la perspectiva de algunos miembros del colectivo son considerados también como presos políticos.

\section{El Tropezón}

La Villa está ubicada en la intersección de las Avda. Cárcano y Avda. Don Bosco, en la zona oeste de la ciudad de Córdoba. A partir de los años 2008-2009 se realizó el traslado de los vecinos de Villa Costa Canal Don Bosco a las que serían sus nuevas viviendas en el barrio Villa Aspacia, en el marco del proceso denominado por el Gobierno de la Provincia como de "erradicación de villas y reubicación en barrios-ciudad", que en Córdoba se extendió entre los años 2003 y 2008; por otro lado comenzóla construcción del megaemprendimiento inmobiliario de nueve torres denominado Alto Villasol ${ }^{13}$, por parte de la empresa Gama S.A., sobre el arroyo El Infiernillo.

A GAMA y al Hipermercado Walmart que está ubicado frente al mismo, se fueron sumando diversos emprendimiento inmobiliarios en la zona y desde el 2011 hasta 2013 la construcción del Nudo Vial más importante de la Provincia — con ingreso a la Córdoba desde ciudades como Carlos Paz o La Calera-, en pleno territorio ocupado desde hace 50 años por la propia Villa El Tropezón ${ }^{14}$.

13 En este apartado, sigo la historización que realizaron Aldo Morales y Emilio Seveso.

14 En línea: http://www.lavoz.com.ar/ciudadanos/el-tropezon-el-nudo-vial-ya-esta-habilitado-y-manana-lo-inauguran. Véase también: http://www.lavoz.com.ar/editorial/los-peatones-la-prioridad (8/2/2016); http://www. lavoz.com.ar/ciudadanos/los-peatones-el-ultimo-eslabon-al-planificar (5/2/2016) y http://www.lavoz.com.ar/ ciudadanos/tropezon-sin-pasarelas-la-caminera-cuidara-los-peatones (30/12/2015), dondequeda expuesto que los habitantes de la villa no sólo fueron ignorados en lo que hace a sus demandas habitacionales, sino hasta en sus 
En adyacencias de los terrenos habitados por esta comunidad se comenzó a generar un fuerte proceso de gentrificación urbana, tanto pública como privada, como lo ejemplifica — frente a la Villa - el mega emprendimiento habitacional Alto Villasol.

En la página de GAMA S.A., Alto Villasol se define en los siguientes términos: "Barrio cerrado en Altura. 9 torres distribuidas en un predio de 5 hectáreas con características propias y el privilegio de vivir con un estilo superior, el "estilo Gama"15. Fue la construcción de estas nueve torres la primera de una serie de obras que agravaron en los últimos años la crisis habitacional vivida por los vecinos de Villa El Tropezón. Realizada sobre el cauce del arroyo El Infiernillo, y sin haberse hecho el correspondiente encauzamiento del mismo - obra exigida por la Provincia al momento de autorizar la construcción de las torres para evitar desbordes de agua-, generó una gran inundación en El Tropezón en 2010, transformando el sector oficialmente en zona inundable. Finalmente, la Provincia absorbió el costo de la obra hidráulica, sin dar una solución definitiva a los problemas de inundaciones de los vecinos.

Figura 2: Nudo vial “El Tropezón”, desde la perspectiva de los vecinos del asentamiento

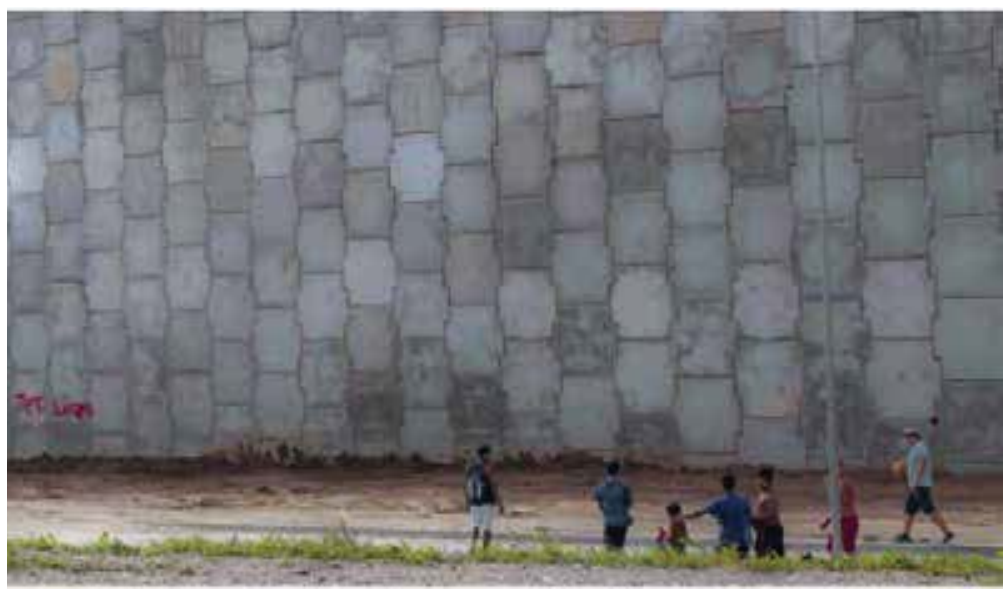

Figura: Lic. Aldo Esteban Morales.

posibilidades de desplazarse. Lo que importa en la convergencia de intereses Estado/Mercado es la circulación de vehículos, sujetos (otro tipo de sujetos) y mercancías; la fluidez y la velocidad de esos cuerpos y objetos que se desplazan desde el oeste (zona de nuevas urbanizaciones y emprendimientos inmobiliarios para las clases medias y medias altas en barrios cerrado y en construcciones en altura) hacia el centro de la ciudad. Hubo muertes de los vecinos por accidentes de tránsito y es a partir de esto que se construyen las pasarelas.

15 Sugerimos visitar la siguiente página web y observar el plano de esta urbanización: http://gama-sa.com/altovillasol/. En esta "segunda naturaleza", las torres de cemento se nominan como los cerros de la provincia: torre 1, Aconcagua;2,Champaquí, 3, Catedral;4,Pan de Azúcar,5, Bastión,6,Cerro Colorado; 7, Tronador, 8, Los Gigantes y 9: Uritorco. 
Pero además de las inundaciones, las obras acarrearon otra serie de graves problemas a los pobladores de El Tropezón: el agrietamiento e incluso derrumbe de viviendas, debido a las intensas vibraciones generadas por las maquinarias; agravamiento de las inundaciones producidas desde el 2010, esta vez por el desvío de los desagües de lluvia para la construcción de la nueva obra, y en 2014 el corte de suministro de agua al sector conformado por las 78 viviendas del extremo más próximo a la obra vial.

En 2009, el entonces Ministro de Desarrollo Social de la Provincia, Carlos Massei, manifestaba que el proceso de reubicación de villas en sectores de la ciudad ajenos a los vecinos finalizaba, comenzando un nuevo proceso de urbanización de villas en el mismo sector de residencia, a través del programa denominado ProMeBa (Programa Mejoramiento de Barrios) ${ }^{\mathbf{1 6}}$. Pero a la situación deEl Tropezón continuaron planteándola como una excepción: al haberse comenzado a considerar el sector como "zona inundable", desde el Ministerio de Desarrollo Social (durante las gestiones de Schiaretti y De La Sota hasta la actualidad) se utilizó este hecho como argumento para establecer la prioridad de "reubicar" a los vecinos de la Villa en otros barrios de la ciudad.

En lucha por los impactos de las intervenciones públicas y privadas antes referidas, los habitantes de la villa se reúnen en asamblea y han conformado una comisión vecinal (2013), para interactuar con ministros, técnicos y demás representantes del Ministerio de Desarrollo Social. En ese año se firmó un acta acuerdo en la cual el gobierno se comprometía a construir 78 viviendas en zonas aledañas a la Villa, reubicando allí las familias que se encontraban más próximas a las obras del nudo vial ${ }^{17}$. En ese mismo acto se oficializó el censo realizado en El Tropezón por el propio ministerio, en el cual identificó que en la Villa vivían en esa fecha 289 familias — número que los vecinos siempre reclamaron como menor al real, en función de lo relevado mediante el trabajo de campo de Morales-. A fines de 2014 se conformó la segunda comisión vecinal, que llego a un acuerdo con el Ministerio de Desarrollo Social para la relocalización de 50 familias a nuevas casas construidas en el Barrio Héroes de Malvinas, a especificar a lo largo del año 2015. Producto de este acuerdo y del anterior, del total de las familias censadas sólo habrá acciones concretas para 128 de ellas; las 161 restantes no tienen respuesta.

\section{EL HORIZONTE DE CAMBIO SOCIAL}

En función de lo descripto en el apartado anterior, afirmamos que el marco ideológico que organiza y orienta las demandas se distingue por un macro rasgo y dinámica: la reacción. La primera definición que han seguido los colectivos

16 Véase: Mazur, M. E., Morales, A. E. y Pardo, D. (2012).

17 A partir de la firma de este convenio, comenzaron a fines de 2013 las obras de construcción del nuevo Nudo Vial, a cargo de la UTE conformada por Roggio y Electroingeniería. 
en su proceso de conformación fue una vía negativa, expuesta como expresividad social construida en las consignas "Ya basta", "Paren de demoler", "No a la demolición". La primera voluntad se orienta a detener, a limitar el avance de grupos económicos sobre los escenarios barriales; escenarios que operan también como el marco de la acción posible en la que disputan los colectivos. El barrio es así el primer espacio - o el único, en algunos casos- en el que se territorializa la lucha. Las expectativas se enmarcan en mantener la "vida barrial" - significante en disputa, en función de las transformaciones que va adquiriendo la acción colectiva y sus resultados-.

Si consideramos una perspectiva diacrónica que amplía el foco a otros colectivos que han luchado con relación al derecho al hábitat en la ciudad cordobesa, es posible generar pistas que ayuden a la comprensión de este primer encuadre de la experiencia de disputa, por parte de los actores que habitan barrios cercanos al centro.

En la ciudad de Córdoba, durante la década del ‘90, algunos sujetos de las clases subalternas lograron organizarse en cooperativas y participar de una instancia no sólo territorial sino sectorial en materia de demanda/concertación sobre el hábitat:La Unión de Organizaciones de Base por los Derechos Sociales. La UOBDS nucleaba a cooperativas de vivienda y participaba de la Mesa de Concertación de Políticas Públicas junto a ONGsy representantes del gobierno provincial y municipal. A diferencia de otras ciudades, en Córdoba no es habitual la vía cooperativa como modalidad de acceso a la vivienda; más bien ésta se resuelve de manera individual/familiar; por lo cual también fue innovadora esta forma de abordaje en materia de hábitat. Esa experiencia participativa y de gestión fue socavada por la crisis provincial de 1997 y no fue reeditada por el nuevo gobierno provincial de otro signo político en 1999. Las nuevas respuestas habitacionales que se generaron desde 2003 ya no convocaron a los colectivos organizados en cooperativas en ninguno de los momentos que conforman el proyecto de construcción de viviendas (planificación, ejecución, evaluación), sino que mediante el plan conocido como "Mi Casa, Mi Vida" (BID) - replicado en el continente- interpelaban a cada familia para acceder al "techo de tus sueños" (slogan oficial del plan) en unidades habitacionales de 6 por 7 metros cuadrados, generalmente por fuera del anillo perimetral de la ciudad. Desde estelugar, los barrios-ciudad pueden ser interpretados comola resultante de un momento del largo proceso de desgaste/ o activa destrucción de los colectivos que demandaban con relación al hábitat en términos de cuestión social.

A pesar de las especificidades en las transformaciones y disputas en los barrios que hemos presentado, éstas pueden ser leídas como fenómenos convergentes con otras manifestaciones del embellecimiento estratégico y la segregación clasista que está reordenando la ciudad de Córdoba desde 2003. Con los pobres afuera, fragmentados y encerrados en los barrios-ciudad, hoy asistimos a la operatoria de grupos económicos en acuerdo con los gobiernos 
(tanto en materia de cambio en las reglamentaciones como en la realización/ adjudicación a esos mismos grupos de obras públicas) avanzando sobre los barrios más próximos al centro. La convergencia de intereses entre ambos actores (estado/mercado) en el rediseño de la ciudad de Córdoba, imprime y adelanta a los vecinos la potencia del impulso desarrollista, como reorganización del espacio en función del capital.

Las intervenciones, demoliciones y regulaciones de las maneras de vivir y estar juntos confluyen en el intento por unir cuerpos a espacios permitidos, - desalojarlos de otros-intentos que no suceden sin resistencias, tal como hemos visto. Estas resistencias se pueden activar porque los miembros de los colectivos de los barrios pericentrales - a diferencia de los pobladores de barrio/ciudad-cuentan con experiencia de lucha en otros espacios de demanda: laborales, estudiantiles (a nivel medio, universitario) que se direccionan hacia el barrio: la suma de vivencias en este lugar, la vida-vivida como recuerdo, presente conflictivo y futuro a cambiar de dirección, los usos sociales de los espacios, son los que están en tensión. Y esas sensibilidades anudan sus narraciones a una idea de barrio-pueblo: con autonomía, identidad, historia y plasticidad.

Lo antes dicho muestra también las ambivalencias o alternancias con relación a las demandas hacia los gobiernos que actualizan estos colectivos: se solicitan cambios/mantenimiento de reglamentaciones, declaraciones que reconozcan el valor patrimonial de algunas edificaciones, pero a la vez, el gobierno aparece como antagonista, cuando se evidencia que durante estos años de democracia, aparece cada vez más claro - y se expone en términos de crisis de representatividad de los partidos- la representación de quienes gobiernan de los intereses de grupos económicos vinculados a la transformación de la ciudad, en desmedro de la representación de las demandas de los ciudadanos. De allí que exista un ir y venir o si se quiere, una configuración confusa con relación al espacio público estatal y sus representantes. Al punto de que algunos colectivos, como la Multisectorial, se hayan propuesto - y logrado- disputar y ganar el Centro Vecinal Barrial.

Pero esta mirada diacrónica que estamos desarrollando no sólo se centra en las transformaciones de la acción colectiva "que se ve", sino que sostenida en una concepción materialista de la cultura que lo que indaga es el marco de expectativas sobre la acción, también interroga el marco ideológico que organiza lo visible, lo no visible y los marcos de lo imaginable y lo no inimaginable del quehacer colectivo, parafraseando la noción žižekeana de ideología (2003).

Un rasgo de este marco, en función de la experiencia de demanda de clase de estos colectivos, es la inscripción de los conflictos en el campo legal: de este modo los ejes de disputa se centran en mantener o cambiar leyes. Oen términos de Žižek nuevamente, la inscripción de los conflictos se concreta en el marco demócrata formal de formulación/tratamiento y resolución. La mayor parte de la conflictividad urbana relevada se instancia en el campo de puja política con 
las instituciones y organismos de gobierno (local, provincial y en algunos casos nacional, como con la expropiación del cine-teatro La Piojera en Alberdi) y la respuesta, es el out/put como mantenimiento, transformación o emergencia de leyes y ordenanzas.

Esta subjetividad hegemónica, asociada a la representación del conflicto como objeto de abordaje desde el reconocimiento/ la ampliación de derechos, tiene limitaciones: sabemos que el Derecho es también resultante de un estado de las clases y de la lucha y horizonte de lucha de clases, ya señalado por Marx y también —-más próximo a nuestro presente— por Daniel Bensaïd. Este autor encuentra en los escritos de Marx la anticipación de las consideraciones que refieren a la fuerza y la violencia escritas en la filigrana de la ley.

Entre dos derechos iguales, escribirá Marx en El Capital, es la fuerza la que decide". Entre dos derechos consuetudinarios contrarios, es también la fuerzala que zanja la cuestión. Se puede seguir así, en la historia social, el hilo conductor que relaciona el antiguo derecho consuetudinario, o 'la economía moral' de los pobres, con los derechos a la vida, a la existencia, al empleo, a los ingresos, a la vivienda, oponibles a los derechos de la propiedad privada (en Bensaï, 2011, p. 26).

Desde aquí aparece como significante la posibilidad de rechazar/cuestionar el acto de codificarse a sí mismos en el marco de las convenciones del lenguaje político del Estado - particularmente los discursos de "derechos" y "ciudadanía" - posibilidad que tímidamente y con interrupciones esbozan algunos colectivos. Sabemos con Rancière y mucho antes con Marx que lo que constituye la potencial fuerza disruptiva de las experiencias de disputa, es el ejercicio de comenzar a balbucear formas/contenidos que "desacuerdan" con los supuestos - de clase - sobre los que se construye y legitima la posición de ciudadanía formal. Ese intersticio es el "entre" que hace posible la distancia crítica respecto a los principios de visión y división que propone el "pensamiento de Estado". La identificación de este "entre" permite señalar algunos aspectos extensivos a abordar con relación al horizonte de lo posible/imaginable para la acción colectiva: en nuestro caso, lo que queda fuera de lo tematizable en las luchas y organizaciones de vecinos, es una referencia más estructural sobre los modelos socio-económicos a partir de los cuales deviene esta forma ciudad y esta forma de transformación del barrio.

Es innegable que se abre un espacio habilitante para nuevas demandas de identidad y subjetividad asociadas al quehacer de los grupos o colectivos, cuyo primer rasgo positivo es sacar al ciudadano de su vivienda y cierto corrimiento de la valoración de la acción individual como la manera de resolver las necesidades. Pero lo anterior también se enmarca en la siguiente creencia como organizadora de quehacer colectivo: el alto grado de aceptación del capitalismo en su operatoria a escala planetaria en generaly en estos territorios en particular 
como algo dado/incuestionabley, como consecuencia, la reducción delos marcos de la acción colectiva pensable/imaginable a un espectro de constricción/ libertad de prácticas, fundados en esta creencia.

Desde estas consideraciones, la forma ciudad y la forma barrio materializan el creciente proceso de dominio del valor del cambio sobre el escenario urbano cordobés, en desmedro delos valores de uso plurales y heterogéneos a partir delos cuales los sujetos y colectivos van tramando significados específicos sobre la vida barrial. Esa forma manifiesta el despliegue de la "revalorización" de la inversión pública y privada e implica la generación de nuevos capitales y nichos de acumulación; se trata de la nueva configuración de la ciudad en tiempos de la planetarización del capital y la reconversión del Estado nacional, provincial y municipal.

Mientras que el aumento del valor de cambio de la tierra entendida como mercancía tiende a crecer, los actores denuncian que esto sucede a costas de la calidad de vida de los habitantes del barrio. Frente a la especulación y a la conservación que orientan las políticas estatales y privadas, los habitantes de estos barrios intentan detener el avance desarrollista, para habitarlos en la espacio/ temporalidad de barrios-pueblo o barrios-obreros que han sido, conservar sus vínculos con el espacio desde la fijación identitaria con un modo de vivir y un pasado común. Hemos visto que muchos reclamos hacen énfasis en el valor de uso de la tierra, la vivienda y los espacios comunes. O para decirlo en otros términos: mientras las políticas hegemónicas intentan desalojar los espacios -y vaciar las calles- para luego transformarlos en un "patrimonio-museo" que no puede ser usado por los vecinos sino por nuevos habitantes de otras clases, las organizaciones reclaman el reconocimiento del "patrimonio-vida" enlazado a las identidades colectivas, narraciones y usos que los actores reconocen y valoran.

En esta línea y como hemos tematizado sobre las luchas migrantes (Domenech y Boito, 2016, en prensa), una de las maneras de disputar más estructuralmente las explicaciones sobre el presente urbano y planificar el quehacer desde la disputa barrial, es recordar la definición que Sandro Mezzadra (2014) concreta con relación al capital, quien también volviendo a Marx, afirma:

\footnotetext{
El capital, como vimos, no es una "cosa", sino "una relación social mediada por cosas". Es importante apreciar el doble significado que asume aquí el término "relación", de fundamental importancia en toda la obra de Marx. La existencia misma dela relación sintetiza, por un lado, una serie de condiciones de sujeción que hacen que determinados sujetos sevean compelidos a ocupar una posición subordinada. Por otro lado, sin embargo, no teniendo la fijeza de una "cosa", la relación de capital es abierta a su contestación, subversión y transformación (2014, p. 135).
}

De esta manera, el capital como relación social es producto y a la vez condición de producción del poder impersonal del reino de la mercancía; pero es tensiva. Por un lado, la existencia misma de la relación indica condiciones de 
sujeción; pero si al mismo tiempo la relación de capital es abierta a su contestación, subversión y transformación es en el "entre" - en el punto de intersección entre los dispositivos de sujeción y las prácticas de subjetivación-donde pueden emerger figuras de subjetividad (Mezzadra, 2014) que van tensionando y produciendolos decires de otros mundos posibles, en los términos de Rancière.

Las luchas y disputas que hemos referido - que se despliegan de forma desigual en los territorios estudiados-, expresan algún grado de variedad con relación a las modalidades de contestación, subversión y transformación, más acá o más allá de su inscripción en el paradigma hegemónico de los derechos antes citado. Si la dominancia del capitalismo se expresa en la centralidad de la lógica de la equivalencia y la consecuente traductibilidad (¿de todo?) asociada al valor de cambio, las prácticas de los sujetos que expresan valores de uso asociados a espacios, lugares y relaciones, van señalando procesos, prácticas y medios de comunicación desde los cuales se van construyendo los modos populares de relacionarse y las maneras que tienen los vecinos de estar/sentirse juntos, en la interrogación que concreta sobre las experiencias comunicativas de nuestro presente, el pensador Jesús Martín-Barbero, al que referimos en el cierre de nuestro trabajo. conflictos barriales/urbanos y lógicas de acción que ponen en acto los colectivos; luego hemos identificado - a partir de las recurrencias observadas-ciertos núcleos de sentido organizadores del horizonte de cambio social y hemos realizado interpretaciones sobre las posibilidades de libertad y de restricción de la acción a las que se asocian. En este punto final del recorrido descriptivo y analítico expuesto aparece como evidente la significación tanto epistémica como política de retomar algunas consideraciones que Jesús Martín Barbero (2015) expresa cuando se pregunta por las formas de estar juntos en los escenarios urbanos contemporáneos. En el artículo titulado “¿Desde dónde pensamos hoy la comunicación?", muestra que la interrogación por la comunicación es aquella que indaga sobre las formas de estar juntos de las cuales participan y a la vez van construyendo las mayorías en las ciudades.

En el escrito hemos subrayado el marco de tendencias que - con creciente intensidad-mediatizan y mercantilizan la vida social en la ciudad, cuya resultante fundamental es la fragmentación y la segregación de las clases; resultante que aparece en los conflictos urbanos abordados y que no puede evitar ser leída como la manifestación de la operatoria del capitalismo - en su forma de finanzas y de construcción - y de las formas de despojo asociadas, en su impacto sobre espacios y cuerpos según clase. 
Siguiendo con la perspectiva de Barbero, la comunicación — conformada por procesos, prácticas y medios a través de los cuales se materializan las maneras de "estar juntos" - abre posibles del hacer con otros, pero también señala el peso de los muros materiales y de los marcos de los horizontes de cambio en tanto distancias - entre clases-y fragmentaciones - en el hacer con otros-. Es por esto que así como hay nuevas formas de estar juntos - por ejemplo, estar conectados vía las nuevas tecnologías (lo que mínimamente hemos referido sobre páginas web y los "problemas" de comunicación para potenciar la participación del "vecino") - a la vez aparecen crueles formas de separación novedosas o viejas modalidades que se reeditan: la pared del nudo vial ante los ojos de los habitantes de El Tropezón que mostramos mediante el registro fotográfico, expone la materialidad de las barreras materiales y sensibles que configuran la experiencia de los sujetos, como expresión de la distancia entre estos pobladores y el resto de los cordobeses.

En continuidad con lo referido por Barbero, el autor da cuenta de "los laberintos que comunican los medios con los miedos". Por nuestra parte, hemos señalado que en la ciudad de Córdoba coexisten tendencias a la socio-segregación clasista en el espacio urbano junto a las modalidades de mediatización cultural de la experiencia; para ser más precisos: a partir de las construcción material de muros - como los que hemos indicado-es factible producir una especie de pantalla para no ver /a ciertas clases/ y proyectar y amplificar los miedos que circulan por los medios, mediante los clásicos mecanismos de des-rostrificación de los pobladores más pobres o sobre-rostrificación/personalización del mal, a partir de la ocurrencia de algunos de violencia urbana (hechos que muestra la violencia subjetiva y los muestra como "sujetos peligrosos" y que en el mismo acto obtura la violencia de todos los días, del muro vuelto "naturaleza" que no permite considerarlos como "sujetos en peligro").

De este modo las formas colectivas de configuración de la experiencia de disputas - en una formación social como la cordobesa, cada vez más socio-segregada y mediatizada culturalmente - vuelven pertinente retornar al temprano señalamiento de S. Hall (1981) sobre los medios "tradicionales" y - en nuestro caso, el nuevo mundo de la red de redes- donde a partir de las transformaciones en el escenario urbano identificadas, se responderán las funciones ideológicas que los caracterizan: suministrando y constituyendo selectivamente el conocimiento social, ofreciendo mapas y códigos que marcan los territorios (geográficos, pero también sociales), brindando contextos explicativos para los acontecimientos y relaciones problemáticas. Funciones que suponen una producción de sentido enfáticamente activa, al converger con las tendencias urbanísticas ya indicadas, y que reinstalan la pertinencia de insistir en la interrogación sobre las maneras de estar juntos/estar separados en el escenario urbano cordobés actual. 


\section{REFERENCIAS}

Benjamin, W. (2005). Libro de los Pasajes. España: AKAL.

Bensaïd, D. (2011). Los desposeídos: Karl Marx, los ladrones de madera y los derechos de los pobres. Buenos Aires: Prometeo Libros.

Boito, M.E., Huergo, J. \& Pereyra, A. (2017, abril). Barrio Güemes contemporáneo: entre lapulsión patrimonialarquitectónica/ciudadanaylacrecienteofertagastronómica turística. En Congreso Internacional de Estudios Urbanos, Universidad Nacional de Quilmes (UNQ), Argentina, 4 al 6 de abril, 2017 (en prensa).

Boito, M. E. \& Pereyra, A. S. (2016, enero-junio). Embellecimiento estratégico en la ciudad de Córdoba: continuidades, tensiones y rupturas en las prácticas del habitar en el barrio Güemes (2000-2014). En Revista de Estudios Socioterritoriales, 19, Centro de Investigaciones Geográficas CIG-Instituto de Geografía, Historia y Ciencias Sociales CIG-IGEHCS CONICET/UNCPBA. En línea: http://www. unicen.edu.ar/content/revista-estudios-socioterritoriales

Boito, M. E. \& Espoz, M. B. (2016 en prensa). Disputas sobre "lo común": políticas de patrimonio, conflictos y haceres colectivos. (Ciudad de Córdoba, 2015), en Lo común en la construcción de la ciudad. Corina Echavarría y Nidia Abatedaga. CONICET, 6/2016 (en prensa).

Boito, M. E. \& Michelazzo, C. (2015). Patrimonio, identidad y espectáculo: una aproximación a las tensiones que conforman el espacio barrial de Alberdi, Córdoba, durante la última década. En Biblioteca digital de UN de Cuyo, 2015

Boito, M. E. \& Salguero Myers, K. (2015a, diciembre). Complejidades y tensiones de la acción colectiva en ciudades socio-segregadas. El caso de barrio Alberdi y San Vicente. Córdoba, Argentina. Publicación en Actas de la exposición en el GT 126, en la XI RAM, Montevideo, Uruguay, 2015.

Boito, M. E. \& Salguero Myers, K. (2015b). Experiencias sociales y transformaciones urbanasenlaciudad deCórdoba, Argentina(2003-2015).Principales dimensiones de la conflictividad yla sensibilidad en una ciudad socio-segregada del Sur Global. Ponencia en Alas Costa Rica, diciembre de 2015.

Boito, M. E. \& Espoz M. B. (2014) (Compiladoras). Urbanismo Estratégico y Separación clasista. Instantáneas de la ciudad en conflicto. Rosario: Puño y Letra.

Debord, G. (1995). La sociedad del espectáculo. Buenos Aires: La Marca.

De Certeau, M. (2000) La invención de lo cotidiano 1. Artes de Hacer, (trad. Alejandro Pescador), México: Universidad Iberoamericana.

Deleuze, G. (1991). Posdata sobre las sociedades de control, en Ferrer, C. (Comp.) El lenguaje literario, 2, Montevideo: Ed. Nordan. 
Domenech, E. \& Boito, M. E. (2016, en prensa). Las "luchas migrantes" en el Sur [de América Latina]: reflexiones críticas desde el enfoque de la autonomía de las migraciones. En Cordero, B.; Mezzadra, S. y Varela, A. (Coordinadores), América latina en movimiento. Migraciones, límites a la movilidad y sus desbordamientos. pp. 190-227 (en prensa).

Harvey, D. (2004) El nuevo imperialismo. Acumulación por desposesión en Socialist Register, Buenos Aires: CLACSO.

Hall, S. (1981). La cultura, los medios de comunicación y los "efectos ideológicos", en Curran, J. et al. (comp.) Sociedad y comunicación de masas, México: Fondo de Cultura Económica.

Levstein, A. \& Boito, M. E. (2009) (Compiladoras). De insomnios y vigilias en el espacio urbano cordobés: lecturas sobre 'Ciudad de mis sueños'. Córdoba: Editorial Jorge Sarmiento Editor, CEA/UE-CONICET.

Martín-Barbero, J. (2015). ¿Desde dónde pensamos la comunicación hoy? Revista Chasqui, 128, sección Tribuna, marzo de 2015, pp. 6-21.

Mazur, M. E., Morales, A. E. \& Pardo, D. (2012). Reflexiones en torno a la participación de los jóvenes en los procesos comunitarios de Villa El Tropezón. Enmarcadas en un contexto de visibilización de acciones colectivas por riesgo de desalojo. Sistematización de Prácticas Pre-Profesionales Contexto Social y Comunitario. Tesis de licenciatura no publicada. Facultad de Psicología, UNC, Córdoba, Argentina.

Mezzadra, S. (2014).LacocinadeMarx.Elsujetoysuproducción. Buenos Aires: Ediciones Tinta limón.

Rancière, J. (1996). El Descuerdo. Política y Filosofía. Buenos Aires: Nueva Visión.

Sennett, R. (1997). Carne y piedra. El cuerpo y la ciudad en la civilización occidental. España: Alianza Editorial.

Žižek, S. (Comp.) (2003). Ideología. Mapa de una cuestión. Buenos Aires: Fondo de Cultura Económica. 


\section{IDENTIFICACIÓN DE LA AUTORA}

María Eugenia Boito. Licenciada en Trabajo Social (ETS-UNC) y en Comunicación Social (ECIUNC). Magíster en Comunicación y Cultura Contemporánea (CEA-UNC) y Doctora en Ciencias Sociales (UBA). Investigadora Adjunta del CONICET y Profesora Asociada del Seminario de Cultura Popular y Masiva (ECI-UNC), Profesora Adjunta Regular de Comunicación y Trabajo Social (ETS-UNC), Profesora a cargo para el dictado del Seminario Electivo no permanente titulado "Ideología, prácticas y conflictos sociales en el escenario urbano cordobés". Facultad de Psicología, UNC. Ciclo lectivo 2016, 2017 y 2018. Directora del Programa -junto a la Dra. María Belén Espoz Dalmasso- titulado "Ideología, prácticas sociales y conflicto (experiencias contemporáneas de / en la ciudad, Córdoba, primera década del siglo XXI)" -CIECS, UNC y CONICET-. En línea: www. ideologiayconflicto.wordpress.com

Las investigaciones que realiza se centran en describir e interpretar los rasgos y las dinámicas de las estructuras de sentir/estructuras de experiencia de los sujetos de las clases subalternas en contextos de mercantilización y mediatización en los que están inscriptos. Una de las dimensiones que caracteriza a esta perspectiva es la identificación de los mecanismos ideológicos que van regulando la percepción sobre lo posible y lo deseable (social), y que marcan y distinguen el horizonte de la acción con otros.

REGISTRO BIBLIOGRAFICO

Boito, María Eugenia (2017). Estar juntos/estar separados en el escenario urbano cordobés actual.

InMediaciones de la Comunicación, 12(1), 215-239. 